\title{
ADECUACIÓN OROFACIAL PARA FACILITAR LA MASTICACIÓN Y DEGLUCIÓN EN NIÑOS CON PCI
}

\author{
Belkis David Parra Reyes ${ }^{8}$
}

Las características de los niños con Parálisis Cerebral (PC), son muy variadas, por tal motivo es importante clasificarlos adecuadamente. Las principales clasificaciones se basan en categorías que consideran: trastorno motor, topografía de la afección, etiología, hallazgos neuroanatómicos, comorbilidades, capacidad funcional y requerimientos terapéuticos.

Dentro de las comorbilidades están los trastornos de la masticación y la deglución, los cuales ocupan un lugar destacado como fuente de morbimortalidad ${ }^{1,2}$. La masticación y la deglución, además de la alimentación son funciones inherentes en la niñez. Por medio de la primera el niño maneja los alimentos de manera segura, eficiente y competente, según su etapa del desarrollo, logrando progresar en peso y talla o mantenerlos ${ }^{3}$, una alteración de esta función define por igual una alteración de la alimentación toda vez que estas implican cualquier dificultad en succionar, morder, masticar, manipular los alimentos en la cavidad oral, controlar la saliva y tragar ${ }^{4,5}$.

La deglución, en segundo término, es un complejo proceso sensoriomotor subdividido en cuatro fases (preoral, oral, faríngea y esofágica), conociéndose su alteración como disfagia ${ }^{4,6}$. La incidencia de esta desviación en la población "normal" alcanza una tasa del $25 \%$ al $45 \%^{7}$, mientras que en niños con algún trastorno del desarrollo aumenta a un rango del $33 \%$ al $80 \%$.

En la PC, los problemas deglutorios se consideran parte de las alteraciones del movimiento y la postura, siendo particularmente frecuentes. Diversas publicaciones informan altos porcentajes de prevalencia de disfagia en dicha condición: $43 \%{ }^{9}, 50 \%{ }^{2}, 90 \%{ }^{1}$ y $99 \%^{10}$.

Los niños pretérmino forman un grupo de riesgo para presentar parálisis cerebral ${ }^{11}$, y son especialmente susceptibles de presentar un trastorno de masticación y deglución, los cuales se manifiestan por una disfunción de la fase preoral, falta de sello labial, movimientos involuntarios del maxilar inferior, incremento del tiempo de contacto entre los labios y la cuchara, reflejo de mordida tónico, intentos múltiples para tragar, aumento del tiempo de tránsito oral, disfunción motora de la lengua, alteraciones mecánicas por arco palatino elevado, retraso del disparo del reflejo deglutorio, exageración del reflejo nauseoso, hipersensibilidad de la cavidad oral, prolongación de la apnea deglutoria en la fase orofaríngea.

8. Doctor en Ciencias de la Educación por la Universidad Nacional de Educación. Magister en Neurociencias por la Facultad de Medicina de la Universidad Nacional Mayor de San Marcos. Especialista en Motricidad Orofacial otorgado por CPAL - CEFAC. Diplomado en Docencia Universitaria/Docencia en Educación superior por la UNE. Licenciado en Tecnología Médica en la Especialidad de Terapia de Lenguaje por la Universidad Nacional Federico Villarreal. Terapeuta de Lenguaje en Rehabilitación Orofacial en síndromes Neurológicos en el Hospital Nacional Guillermo Almenara I. - EsSALUD. Docente en pre-grado y postgrado de diversos cursos especializados sobre Terapia de Lenguaje en la UNFV, UNMSM y UAP. Miembro de la International Association Of Orofacial Myology (IAOM), de la Academia Latinoamericana de Disfunciones Estomatognáticas (ALDE), Miembro fundador de la Academia de Neurociencias del Perú (ANP), de la Asociación Peruana para el Estudio del Dolor (APED), Miembro fundador de la Comunidad de Motricidad Orofacial Latinoamericana (CMOL), Miembro del cuerpo editorial en la calidad de editor de la revista científica de Medicina de Rehabilitación del Departamento de Medicina de Rehabilitación del Servicio de otras Discapacidades y Minusvalías del HNGAI, de la revista CoDaS (Brasil), columnista de la Revista de Ciencia y Cultura ANRA, Miembro de la Comisión de Investigación del Hospital Nacional Guillermo Almenara Irigoyen, Miembro del Instituto de Investigación de la FTM y del comité consultivo interno como docente investigativo invitado por el Vicerrectorado de Investigación de la UNFV. Director y editor de la revista digital de motricidad Orofacial "RevMOf". Director científico de la Comunidad de Motricidad Orofacial Latinoamericana (CMOL). 
La adecuación de la masticación y deglución en niños con PC debe enfocarse integralmente, apoyado en un programa de terapia orofacial específica para las funciones estomatognáticas ${ }^{12}$. Las prioridades de tratamiento se establecen sobre una base de gravedad del problema deglutorio y según las características funcionales y orofaciales que se presentan.

La terapéutica de los ámbitos motor, respiratorio, nutricional y gastrointestinal debe comprender un manejo transdisciplinario, requiriendo de la experiencia de varios especialistas ${ }^{13,14,15}$, a medida que cambian las necesidades del niño ${ }^{5}$. La familia debe estar incluida en el equipo ya que es pieza importante en el proceso de rehabilitación ${ }^{18}$. Las acciones del equipo se orientan a privilegiar la ingesta suficiente para cubrir necesidades nutricionales y de hidratación del niño, utilizando el método de alimentación más seguro y mejor tolerado por él ${ }^{4,13,15}$. Las metas perseguidas en el abordaje, buscan controlar o minimizar el impacto de la disfunción deglutoria, adecuándolas, pese a sus limitaciones y patrones inadecuados ${ }^{5,13,16,17}$.

\section{REFERENCIAS BIBLIOGRÁFICAS}

1. Salghetti A, Martinuzzi A. Dysphagia in cerebral palsy. East Jour Med. 2012; 17: 188-193.

2. Otapowicz D, Sobaniec W, Okurowska-Zawada B, et al. Dysphagia in children with infantile cerebral palsy. Adv Med Sci. 2010; 55(2): 222-227.

3. Arvedson JC. Swallowing and feeding in infants and young children. GI Mot Online. 2006. doi:10.1038/gimo17.

4. American Speech-Language \& Hearing Association, ASHA. ASHA Clinical Topics: Pediatric Dysphagia. Disponible en: http://www.asha.org/PracticePortal/Clinical-Topics/PediatricDysphagia/. Acceso: Noviembre 18, 2013.

5. Arvedson JC. Assessment of pediatric dysphagia and feeding disorders: clinical and instru mental approaches. Dev Disabil Res Rev. 2008; 14(2): 118- 127.

6. Cámpora H, Falduti A. Evaluación y tratamiento de las alteraciones de la deglución. Rev Am Med Resp. 2012; 3: 98-107.

7. Lefton-Greif M, Arvedson JC. Pediatric feeding and swallowing disorders: state of health, po pulation trends, and application of the International Classification of Functioning, Disability, and Health. Sem Speech \& Lang. 2007; 28: 161-165.

8. Lefton-Greif M. Pediatric Dysphagia. Phys Med \& Rehab Clin N Am. 2008; 19: 837-851.

9. Parkes J, Hill N, Platt MJ, et al. Oromotor dysfunction and communication impairments in children with cerebral palsy: a register study. Dev Med Child neurrol. 2010; 52: 1113-9.

10. Calis EA, Veugelers R, Sheppard JJ, et al. Dysphagia in chidren with severe generalized cere bral palsy and intelectual disability. Dev Med Child Neurol. 2008; 50: 625-630

11. Oskoui M, Coutinho F, Dykeman J, et al. An update on the prevalence of cerebral palsy: a systematic review and meta-analysis. Dev Med Child Neurol. 2013; 55(6): 509-519

12. Cámpora H, Durand L, Ané F. Trastornos de la deglución. Arch Neurol, Neurocir Neuropsiq. 2001; 11: 1-8

13. Ministerio de Salud de Chile. Guía clínica: alimentación en niños con dificultades en masticar y deglutir, derivado de alteración del sistema nervioso. Santiago: Minsal, 2010.

14. Farneti D. Disordini della deglutizione nella pratica medica ambulatoriale. Rev SIMG. 2004; 3: 23-27.

15. García C, Lin P, Prado F, et al. Guías clínicas para la alimentación en pacientes con trastorno de la deglución. Neumol Pediatr. 2011; 6(2): 67-71

16. JBI (Joanna Briggs Institute). Identification and management of dysphagia in children with neurological impairments. Best Practice. 2009; 13(1): 5-8.

17. García C, Lin P, Prado F, et al. Guías clínicas para la alimentación en pacientes con trastorno de la deglución. Neumol Pediatr. 2011; 6(2): 67-71. 
Revista Signos Fónicos

Suplemento especial: II Encuentro Americano y I Iberoamericano de Motricidad Orofacial Lima Perú

18. Andrew MJ, Sullivan PB. Feeding difficulties in disabled children. Paed Child Health. 2010; 20(7): 321-326.

19. Miller CK. Updates on pediatric feeding and swallowing problems. Curr Opin Otolaryngol Head Neck Surg. 2009; 17: 194-199. 